\title{
Product Cordial Labeling for Some New Graphs
}

\author{
S K Vaidya (Corresponding author) \\ Department of Mathematics, Saurashtra University \\ Rajkot - 360 005, Gujarat, India \\ E-mail: samirkvaidya@yahoo.co.in
}

\section{M Barasara}

Department of Mathematics, Atmiya Institute of Technology and Science

Rajkot - 360 005, Gujarat, India

E-mail: chirag.barasara@gmail.com

Received: December 16, 2010 Accepted: December 31, 2010 doi:10.5539/jmr.v3n2p206

\begin{abstract}
In this paper we investigate product cordial labeling for some new graphs. We prove that the friendship graph, cycle with one chord (except when $\mathrm{n}$ is even and the chord joining the vertices at diameter distance), cycle with twin chords (except when $\mathrm{n}$ is even and one of the chord joining the vertices at diameter distance) are product cordial graphs. We also investigated middle graph of path $P_{n}$ admits product cordial labeling.
\end{abstract}

Keywords: Cordial Labeling, Product Cordial Labeling, Product Cordial Graph.

AMS Subject classification number: $05 \mathrm{C} 78$.

\section{Introduction}

We begin with finite, connected and undirected graph $G=(V(G), E(G))$ without loops and multiple edges. Here $C_{n}$ and $P_{n}$ denote the cycle and path respectively. For all standard terminology and notations we follow (Gross, J. and Yellen, J., 1999). We will give brief summary of definitions which are useful for the present investigations.

Definition 1.1 If the vertices of the graph are assigned values subject to certain conditions then it is known as graph labeling.

Most of the graph labeling problems have following three common characteristics:

1. a set of numbers for assignment of vertex labels;

2. a rule that assigns a label to each edge;

3. some condition(s) that these labels must satisfy.

Labeled graphs have applications in many diversified fields. A detailed study on variety of applications of graph labeling is reported in (Bloom, G. S. and Golomb, S. W., 1977, p.562-570).

For extensive survey on graph labeling we refer to (Gallian, J.A., 2010). Enough literature is available in printed as well as electronic form on different types of graph labeling. Labeling of graph is the potential area of research and more than 1200 research papers have been published so far in past five decades. According to (Beineke, L. W. and Hegde, S. M., 2001, p.63-75) the concept of graph labeling is a frontier between number theory and structure of graphs.

Definition 1.2 A mapping $f: V(G) \rightarrow\{0,1\}$ is called binary vertex labeling of $G$ and $f(v)$ is called the label of vertex $v$ of $G$ under $f$.

The induced edge labeling $f^{*}: E(G) \rightarrow\{0,1\}$ is given by $f^{*}(e=u v)=|f(u)-f(v)|$. Let $v_{f}(0), v_{f}(1)$ be the number of vertices of $G$ having labels 0 and 1 respectively under $f$ and let $e_{f}(0), e_{f}(1)$ be the number of edges of $G$ having labels 0 and 1 respectively under $f^{*}$

Definition 1.3 A binary vertex labeling of graph $G$ is called cordial labeling if $\left|v_{f}(0)-v_{f}(1)\right| \leq 1$ and $\left|e_{f}(0)-e_{f}(1)\right| \leq 1$. A graph $G$ is cordial if it admits cordial labeling.

The concept of cordial labeling was introduced by (Cahit, I., 1987, p.201-207) and in the same paper he investigated several results on this newly introduced concept.

Some labelings with variations in cordial theme have also been introduced such as prime cordial labeling, A-cordial labeling, E-cordial labeling, H-cordial labeling, Product cordial labeling etc. The present paper is aimed to investigate some results on product cordial labeling in which absolute difference is replaced by product of the vertex labels.

Definition 1.4 A binary vertex labeling of graph $G$ with induced edge labeling $f^{*}: E(G) \rightarrow\{0,1\}$ defined by $f^{*}(e=u v)=$ 
$f(u) f(v)$ is called a product cordial labeling if $\left|v_{f}(0)-v_{f}(1)\right| \leq 1$ and $\left|e_{f}(0)-e_{f}(1)\right| \leq 1$. A graph $G$ is product cordial if it admits product cordial labeling.

(Sundaram, M., Ponraj, R. and Somsundaram, S., 2004, p.155-163) proved that trees, unicyclic graphs of odd order, triangular snakes, dragons, helms and union of two path graphs are product cordial. They also proved that a graph with $p$ vertices and $q$ edges with $p \geq 4$ is product cordial then $q<\frac{p^{2}-1}{4}$. The graphs obtained by joining apex vertices of $k$ copies of stars, shells and wheels to a new vertex are product cordial is proved in (Vaidya, S. K. and Dani, N. A., 2010, p.63-66) while the product cordial labeling for some cycle related graphs is reported in (Vaidya, S. K. and Kanani, K. K., 2010, p.109-116). In the same paper they have investigated product cordial labeling for the shadow graph of cycle $C_{n}$.

There are three types of problems that can be considered in this area.

1. How product cordiality is affected under various graph operations;

2. Construct new families of product cordial graphs by finding suitable labeling;

3. Given a graph theoretic property $\mathrm{P}$, characterise the class of graphs with property $\mathrm{P}$ that are product cordial.

This paper is focused on problems of first two types.

Definition 1.5 The middle graph $M(G)$ of a graph $G$ is the graph whose vertex set is $V(G) \cup E(G)$ and in which two vertices are adjacent if and only if either they are adjacent edges of $G$ or one is a vertex of $G$ and the other is an edge incident with it.

Definition 1.6 The friendship graph $F_{n}$ is one-point union of $n$ copies of cycles $C_{3}$.

Definition $1.7 \mathrm{~A}$ chord of cycle $C_{n}$ is an edge joining two non-adjacent vertices of cycle $C_{n}$.

Definition 1.8 Two chords of a cycle $C_{n}$ are said to be twin chords if they form a triangle with an edge of cycle $C_{n}$.

In present investigations we prove that the friendship graph, cycle with one chord, cycle with twin chords and middle graph of path are product cordial graphs.

\section{Main Results}

Theorem 2.1 $F_{n}$ is product cordial.

Proof: Let $F_{n}$ be the friendship graph with $n$ copies of cycle $C_{3}$. Let $v^{\prime}$ be the apex vertex, $v_{1}, v_{2}, \ldots, v_{2 n}$ be the other vertices and $e_{1}, e_{2}, \ldots, e_{3 n}$ be the edges of $F_{n}$.

Define $f: V\left(F_{n}\right) \rightarrow\{0,1\}$, we consider following two cases.

Case 1: When $n$ is even.

$$
\begin{array}{ll}
f\left(v_{i}\right)=0, & 1 \leq i \leq n \\
f\left(v_{i}\right)=1, & \text { otherwise } \\
f\left(v^{\prime}\right)=1 &
\end{array}
$$

In view of the above labeling pattern we have,

$$
\begin{gathered}
v_{f}(0)=v_{f}(1)-1=n \\
e_{f}(0)=e_{f}(1)=\frac{3 n}{2}
\end{gathered}
$$

Case 2: When $n$ is odd.

$$
\begin{aligned}
& f\left(v_{i}\right)=0, \quad 1 \leq i \leq n \\
& f\left(v_{i}\right)=1, \quad \text { otherwise } \\
& f\left(v^{\prime}\right)=1
\end{aligned}
$$

In view of the above labeling pattern we have,

$$
\begin{aligned}
& v_{f}(0)+1=v_{f}(1)=n+1 \\
& e_{f}(0)=e_{f}(1)+1=\left\lceil\frac{3 n}{2}\right\rceil
\end{aligned}
$$

Thus in each cases we have $\left|v_{f}(0)-v_{f}(1)\right| \leq 1$ and $\left|e_{f}(0)-e_{f}(1)\right| \leq 1$. Hence $F_{n}$ is product cordial. 
Illustration 2.2 Consider the graph $F_{5}$. The product cordial labeling is shown in figure 1 .

Theorem 2.3 Cycle $C_{n}$ with one chord is product cordial except when $\mathrm{n}$ is even and the chord is joining the vertices at diameter distance.

Proof : Let $G$ be the cycle graph with one chord. Let $v_{1}, v_{2}, \ldots, v_{n}$ be the vertices of $G$. Here graph $G$ has $n$ vertices and $n+1$ edges.

Define $f: V(G) \rightarrow\{0,1\}$, we consider following two cases.

Case 1: When $n$ is odd.

Without loss of generality we assure that let the chord is between vertex $\left(v_{1}, v_{i}\right)$ where $3 \leq i \leq\left\lceil\frac{n}{2}\right\rceil$.

$$
\begin{array}{ll}
f\left(v_{i}\right)=1, & 1 \leq i \leq\left\lceil\frac{n}{2}\right\rceil \\
f\left(v_{i}\right)=0, & \text { otherwise }
\end{array}
$$

In view of the above labeling pattern we have,

$$
\begin{gathered}
v_{f}(0)+1=v_{f}(1)=\left\lceil\frac{n}{2}\right\rceil \\
e_{f}(0)=e_{f}(1)=\frac{n+1}{2}
\end{gathered}
$$

Case 2: When $n$ is even.

Without loss of generality we assume that let the chord is between vertex $\left(v_{1}, v_{i}\right)$ where $3 \leq i \leq \frac{n}{2}+1$.

Subcase 1: When chord is between $\left(v_{1}, v_{i}\right)$ where $i=\frac{n}{2}+1$.

In order to satisfy the vertex condition for product cordial graph it is essential to assign label 0 to $\frac{n}{2}$ vertices out of total $n$ vertices. The vertex with label 0 will give rise at least $\frac{n}{2}+2$ edges with label 0 and at most $\frac{n}{2}-1$ edges with label 1 out of total $n+1$ edges of $G$. Therefore $\left|e_{f}(0)-e_{f}(1)\right|=3$. Thus the edge condition for product cordial graph is violated. Hence $G$ is not product cordial.

Subcase 2: When chord is between $\left(v_{1}, v_{i}\right)$ where $3 \leq i \leq \frac{n}{2}$.

$$
\begin{array}{ll}
f\left(v_{i}\right)=1, & 1 \leq i \leq \frac{n}{2} \\
f\left(v_{i}\right)=0, & \text { otherwise }
\end{array}
$$

In view of the above labeling pattern we have,

$$
\begin{gathered}
v_{f}(0)=v_{f}(1)=\frac{n}{2} \\
e_{f}(0)=e_{f}(1)-1=\frac{n}{2}+1
\end{gathered}
$$

Thus in each cases we have $\left|v_{f}(0)-v_{f}(1)\right| \leq 1$ and $\left|e_{f}(0)-e_{f}(1)\right| \leq 1$. Hence Cycle $C_{n}$ with one chord is product cordial.

Illustration 2.4 Consider the cycle $C_{7}$ with one chord. The product cordial labeling is shown in figure 2.

Theorem 2.5 Cycle $C_{n}$ with twin chords is product cordial except when $\mathrm{n}$ is even and one of the chord joining vertices at diameter distance.

Proof: Let $G$ be the cycle graph with twin chords. Let $v_{1}, v_{2}, \ldots, v_{n}$ be the vertices of $G$. Here graph $G$ has $n$ vertices and $n+2$ edges.

Define $f: V(G) \rightarrow\{0,1\}$, we consider following two cases.

Case 1: When $n$ is odd.

Without loss of generality we assume that let the chords are between vertex $\left(v_{1}, v_{i}\right)$ and $\left(v_{1}, v_{i+1}\right)$ where $3 \leq i \leq\left\lceil\frac{n}{2}\right\rceil$. 


$$
\begin{array}{ll}
f\left(v_{i}\right)=1, & 1 \leq i \leq\left\lceil\frac{n}{2}\right\rceil \\
f\left(v_{i}\right)=0, & \text { otherwise }
\end{array}
$$

In view of the above labeling pattern we have,

$$
v_{f}(0)+1=v_{f}(1)=\left\lceil\frac{n}{2}\right\rceil
$$

$e_{f}(0)=e_{f}(1)+1=\left\lceil\frac{n}{2}\right\rceil+1 \quad ; \quad$ when chords are between $\left(v_{1}, v_{i}\right)$ and $\left(v_{1}, v_{i+1}\right)$ where $i=\left\lceil\frac{n}{2}\right\rceil$.

$e_{f}(0)+1=e_{f}(1)=\left\lceil\frac{n}{2}\right\rceil+1 \quad ; \quad$ when chords are between $\left(v_{1}, v_{i}\right)$ and $\left(v_{1}, v_{i+1}\right)$ where $3 \leq i<\left\lceil\frac{n}{2}\right\rceil$.

Case 2: When $n$ is even.

Without loss of generality we assume that let the chords are between vertex $\left(v_{1}, v_{i}\right)$ and $\left(v_{1}, v_{i+1}\right)$ where $3 \leq i \leq \frac{n}{2}$.

Subcase 1: When chords are between $\left(v_{1}, v_{i}\right)$ and $\left(v_{1}, v_{i+1}\right)$ where $i=\frac{n}{2}$.

In order to satisfy the vertex condition for product cordial graph it is essential to assign label 0 to $\frac{n}{2}$ vertices out of total $n$ vertices. The vertex with label 0 will give rise at least $\frac{n}{2}+2$ edges with label 0 and at most $\frac{n}{2}$ edges with label 1 out of total $n+2$ edges of $G$. Therefore $\left|e_{f}(0)-e_{f}(1)\right|=2$. Thus the edge condition for product cordial graph is violated. Hence $G$ is not product cordial.

Subcase 2: When chords are between $\left(v_{1}, v_{i}\right)$ and $\left(v_{1}, v_{i+1}\right)$ where $3 \leq i<\frac{n}{2}$.

$$
\begin{array}{ll}
f\left(v_{i}\right)=1, & 1 \leq i \leq \frac{n}{2} \\
f\left(v_{i}\right)=0, & \text { otherwise }
\end{array}
$$

In view of the above labeling pattern we have,

$$
\begin{gathered}
v_{f}(0)=v_{f}(1)=\frac{n}{2} \\
e_{f}(0)=e_{f}(1)=\frac{n}{2}+1
\end{gathered}
$$

Thus in each cases we have $\left|v_{f}(0)-v_{f}(1)\right| \leq 1$ and $\left|e_{f}(0)-e_{f}(1)\right| \leq 1$. Hence Cycle $C_{n}$ with twin chords is product cordial.

Illustration 2.6 Consider the cycle $C_{8}$ with twin chords. The product cordial labeling is shown in figure 3 .

Theorem 2.7 $M\left(P_{n}\right)$ is Product Cordial.

Proof: If $M\left(P_{n}\right)$ be the middle graph of path $P_{n}$ then let $v_{1}, v_{2}, \ldots, v_{n}$ be the vertices of path $P_{n}$ and $v_{1}^{\prime}, v_{2}^{\prime}, \ldots, v_{n-1}^{\prime}$ be the vertices added corresponding to the edges $e_{1}, e_{2}, \ldots, e_{n-1}$ in order to obtain $M\left(P_{n}\right) . M\left(P_{n}\right)$ has $2 n-1$ vertices and $3 n-4$ edges.

Define $f: V\left(M\left(P_{n}\right)\right) \rightarrow\{0,1\}$, we consider following two cases.

Case 1: When $n$ is even.

$$
\begin{aligned}
& f\left(v_{i}\right)=0, \quad 1 \leq i \leq \frac{n}{2} \\
& f\left(v_{i}\right)=1, \quad \text { otherwise } \\
& f\left(v_{i}^{\prime}\right)=0, \quad 1 \leq i \leq \frac{n}{2}-1 \\
& f\left(v_{i}^{\prime}\right)=1 \text {, otherwise }
\end{aligned}
$$

In view of the above labeling pattern we have,

$$
\begin{gathered}
v_{f}(0)+1=v_{f}(1)=n \\
e_{f}(0)=e_{f}(1)=\frac{3 n-4}{2}
\end{gathered}
$$

Case 2: When $n$ is odd. 


$$
\begin{array}{ll}
f\left(v_{i}\right)=0, & 1 \leq i \leq\left\lfloor\frac{n}{2}\right\rfloor \\
f\left(v_{i}\right)=1, & \text { otherwise } \\
f\left(v_{i}^{\prime}\right)=0, & 1 \leq i \leq\left\lfloor\frac{n}{2}\right\rfloor \\
f\left(v_{i}^{\prime}\right)=1, & \text { otherwise }
\end{array}
$$

In view of the above labeling pattern we have,

$$
\begin{gathered}
v_{f}(0)+1=v_{f}(1)=n \\
e_{f}(0)-1=e_{f}(1)=\left\lfloor\frac{3 n-4}{2}\right\rfloor
\end{gathered}
$$

Thus in each cases we have $\left|v_{f}(0)-v_{f}(1)\right| \leq 1$ and $\left|e_{f}(0)-e_{f}(1)\right| \leq 1$. Hence $M\left(P_{n}\right)$ is product cordial.

Illustration 2.8 Consider the graph $M\left(P_{7}\right)$. The product cordial labeling is shown in figure 4 .

\section{Concluding Remarks}

It is very interesting to investigate whether or not a graph or graph families admit particular type of labeling. Here we investigate four new families of product cordial graphs. To derive similar results on other graph families is an open area of research.

\section{Acknowledgment}

The authors are highly thankful to the anonymous referees for constructive suggestions and comments.

\section{References}

Beineke, L. W. \& Hegde, S. M. (2001). Strongly Multiplicative graphs, Discuss. Math. Graph Theory, 21, 63-75.

Bloom, G. S. \& Golomb, S. W. (1977). Application of Numbered Undirected Graphs, Proc. of IEEE, 65, 562-570.

Cahit, I. (1987). Cordial Graphs: A weaker version of graceful and harmonious graphs, Ars Combin., 23, $201-207$.

Gallian, J. A. (2010). A dynamic survey of graph labeling, The Electronics Journal of Combinatorics, 17 (\#DS6).

Gross, J. \& Yellen, J. (1999). Graph theory and its applications. CRC Press.

Sundaram, M., Ponraj, R. \& Somsundaram, S. (2004). Product cordial labeling of graphs, Bull. Pure and Applied Sciences(Mathematics and Statistics), 23E, 155-163.

Vaidya, S. K. \& Dani, N. A. (2010). Some New Product Cordial Graphs, Journal of App. Comp. Sci. Math., 8(4), 62-65. Vaidya, S. K. \& Kanani, K. K. (2010). Some Cycle Related Product Cordial Graphs, Int. J. of Algorithms, Comp. and Math., 3(1), 109-116.

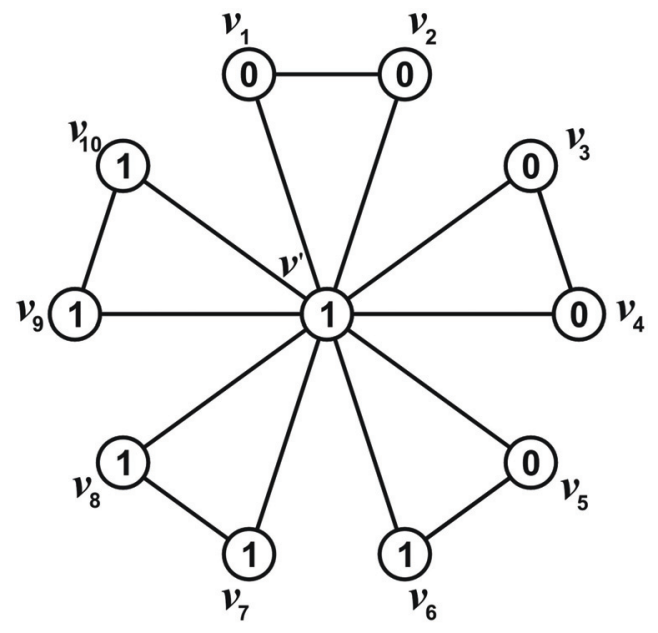

Figure $1 . F_{5}$ with product cordial labeling. 


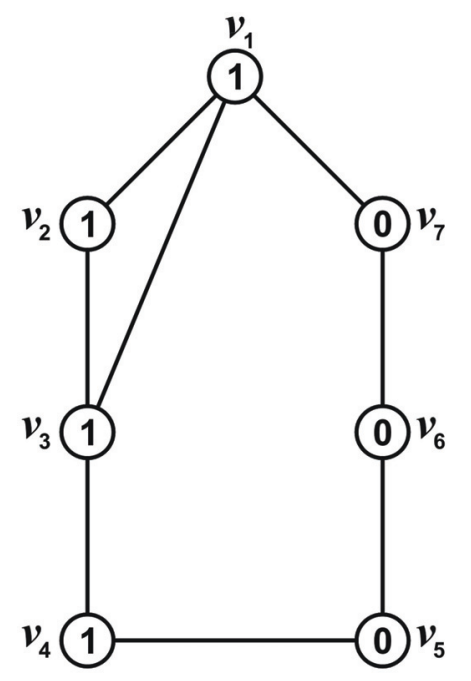

Figure 2. $C_{7}$ with one chord and its product cordial labeling.

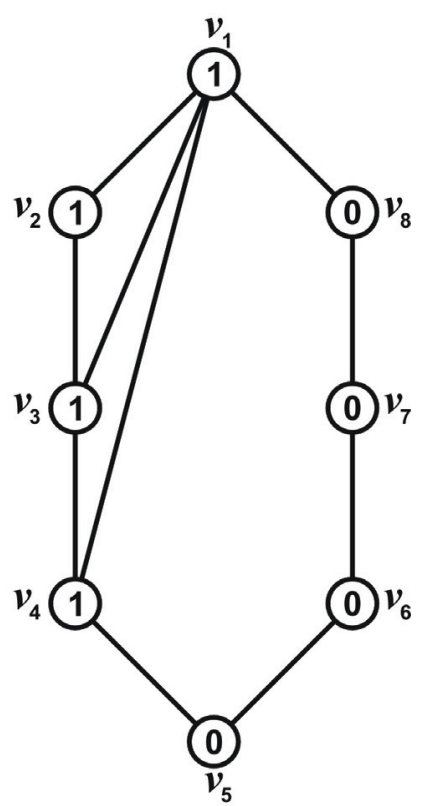

Figure 3. $C_{8}$ with twin chords and its product cordial labeling.

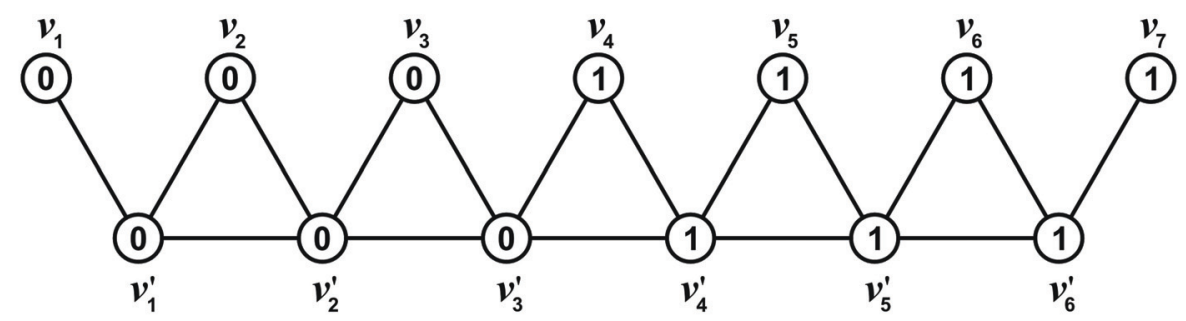

Figure 4. $M\left(P_{7}\right)$ with product cordial labeling. 